\title{
A Case of Pseudoaneurysm of the Internal Carotid Artery Following Endoscopic Endonasal Pituitary Surgery: Endovascular Treatment with Flow-Diverting Stent Implantation
}

\author{
Ali Karadag', Burak Kinali ${ }^{1}$, Omer Ugur ${ }^{2}$, Ismail Oran ${ }^{3}$, Erik H. Middlebrooks ${ }^{4}$, Mehmet Senoglu ${ }^{1, *}$
}

\begin{abstract}
Internal carotid artery (ICA) pseudoaneurysm is a rare complication of endoscopic endonasal surgery occurring in $0.4-1.1 \%$ of cases. Pseudoaneurysms can subsequently result in other complications, such as subarachnoid hemorrhage, epistaxis, and caroticocavernous fistula with resultant death or permanent neurologic deficit. In this case, we illustrate endovascular treatment with a flow-diverting stent for an ICA pseudoaneurysm after endoscopic endonasal surgery for a pituitary adenoma in a 56-year-old male. Surgery was complicated by excessive intraoperative bleeding and emergent $\mathrm{CT}$ angiography confirmed an iatrogenic pseudoaneurysm on the anteromedial surface of the ICA. The pseudoaneurysm was treated endovascularly with flow-diverting stent implantation only. Follow-up CT angiography after three months demonstrated occlusion of the pseudoaneurysm.
\end{abstract}

\section{KEYWORDS}

pituitary; injury; pseudoaneurysm; endovascular; flow diverting stent

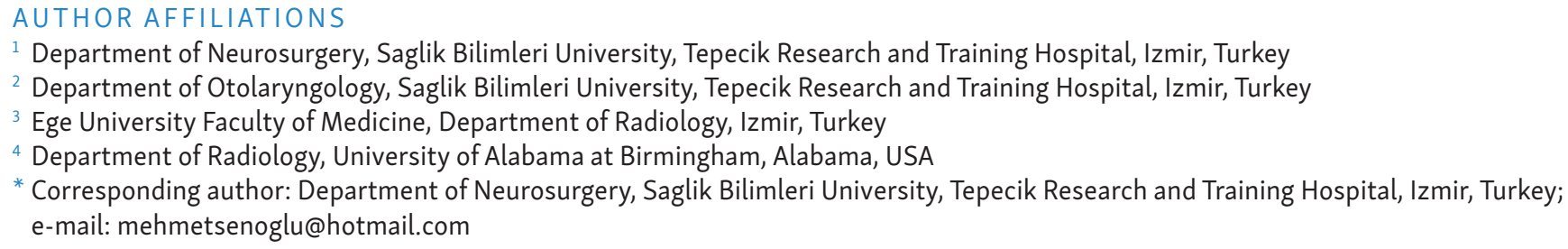

Received: 5 November 2016

Accepted: 28 April 2017

Published online: 5 October 2017

Acta Medica (Hradec Králové) 2017; 60(2): 89-92

https://doi.org/10.14712/18059694.2017.100

(C) 2017 The Authors. This is an open-access article distributed under the terms of the Creative Commons Attribution License (http://creativecommons.org/licenses/by/4.0), which permits unrestricted use, distribution, and reproduction in any medium, provided the original author and source are credited. 


\section{INTRODUCTION}

Internal carotid artery (ICA) pseudoaneurysm is a rare complication of endoscopic endonasal pituitary surgery (1). The incidence of pseudoaneurysm following endoscopic endonasal pituitary surgery has been reported as 0.4-1.1\% (1). Complications of ruptured pseudoaneurysm include subarachnoid hemorrhage, caroticocavernous fistula, or epistaxis, any of which could result in death or permanent disability (2). While risks can be minimized by a thorough knowledge of local anatomy and pre-operative imaging, this information cannot completely eliminate the risk of arterial injury (3).

In the event of unexpected massive bleeding during endonasal surgery, immediate control of hemorrhage is the priority, often achieved by packing (4). Once hemostasis is achieved, CT angiography or digital subtraction angiography (DSA) can assist in identifying underlying arterial injury as a potential cause of hemorrhage. Traditionally, emergency surgical ligation of the ICA is used in these injuries (3). Ligation methods used in ICA injury can cause serious complications, including stroke and death $(3,5)$. With the continued development of endovascular techniques, the potentially debilitating or fatal complications seen after traditional treatment methods suggests endovascular treatment as a potentially better alternative (5). In this study, we report a case of ICA pseudoaneurysm developing after endoscopic endonasal surgery with safe and effective treatment with a flow-diverting stent, thus allowing preservation of the parent artery and occlusion of the iatrogenic pseudoaneurysm.

\section{CASE REPORT}

A 56-year-old male was admitted to the hospital with decreased vision and bitemporal hemianopsia. At the time of admission, the vital signs were: arterial blood pressure of 120/75 mmHg, pulse rate of 80 beats per minute (bpm), respiratory rate of 20 breaths/minute, and body temperature of $36.2^{\circ} \mathrm{C}$. The patient was cooperative and oriented to time, place, and person. There was no weakness present. Laboratory results showed: fasting glucose of $133 \mathrm{mg} / \mathrm{dl}$, prolactin of $40.93 \mathrm{ng} / \mathrm{ml}$. Other laboratory results were in normal range. Magnetic resonance imaging (MRI) with contrast revealed a mass extending from within the sella into the suprasellar space measuring $35 \times 25 \mathrm{~mm}$. The mass caused significant compression on the optic chiasm, hypothalamus, and anterior communicating arteries (Figure 1). There was no definite invasion into the cavernous sinus by MRI.

After appropriate preparations had been made, the patient was taken to the operative room for an endoscopic endonasal approach for mass resection; however, excessive bleeding was encountered (Figure 2), and the operation was aborted. Intraoperative nasal packing was performed to control the hemorrhage (Figure 3). Estimated blood loss was $450 \mathrm{cc}$, but the patient remained hemodynamically stable with no need for blood transfusion. Computed tomography (CT) angiography was performed emergently, which showed no aneurysm or other vascular abnormality. After observation for several days, the patient was discharged and scheduled for completion of the operation one month later.
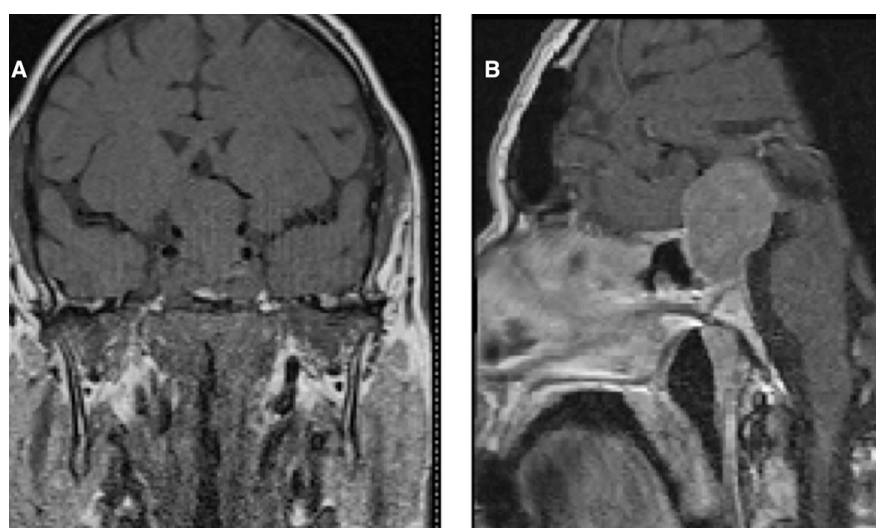

Fig. 1: (A) Coronal non-contrast T1-weighted and (B) sagittal postcontrast T1-weighted MRI show an enhancing mass extending from the sella into the suprasellar space most suggestive of a pituitary macroadenoma.
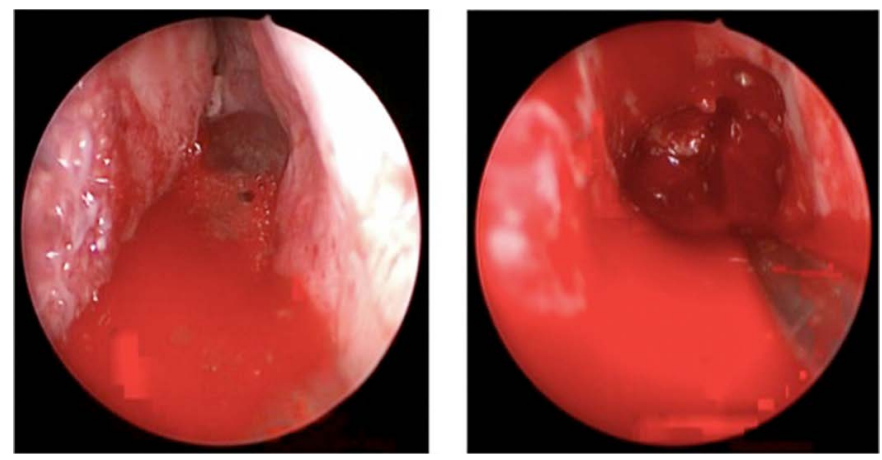

Fig. 2: These pictures show the massive bleeding from the internal carotid artery during the surgery
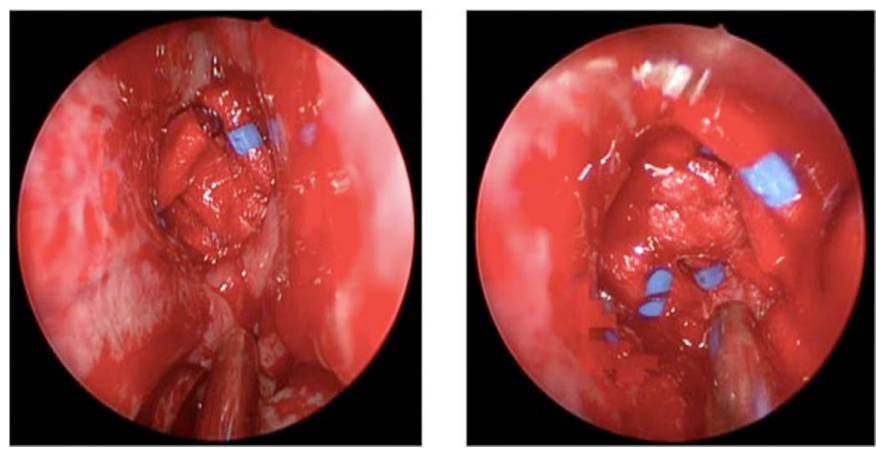

Fig. 3: The pictures show the compress for stopping the bleeding.

During the second operation, excessive bleeding was again encountered and the operation again aborted. At this point, the adenoma was almost completely evacuated (Figure 6). Due to continued concern of vascular injury, a cerebral DSA was performed and did reveal a pseudoaneurysm of the cavernous segment of the left ICA. Decision was made to manage the iatrogenic pseudoaneurysm by endovascular approach. The patient was loaded with $450 \mathrm{mg}$ of clopidogrel and $100 \mathrm{mg}$ of aspirin four hours prior to the endovascular operation. Since this lateral wall pseudoaneurysm was too small to insert coils safely, we placed 
a $3.5 \times 20 \mathrm{~mm}$ flow-diverting stent (Pipeline, Ev3-Covidient, USA) in the parent artery across the pseudoaneurysm without complication (Figure 4). The patient was kept under dual antiaggregant treatment (75 mg clopidogrel and $100 \mathrm{mg}$ aspirin, daily) for three months. Follow-up CT angiography obtained after three months showed the complete exclusion of the iatrogenic pseudoaneurysm from the circulation (Figure 5). There was no neurologic deficit.

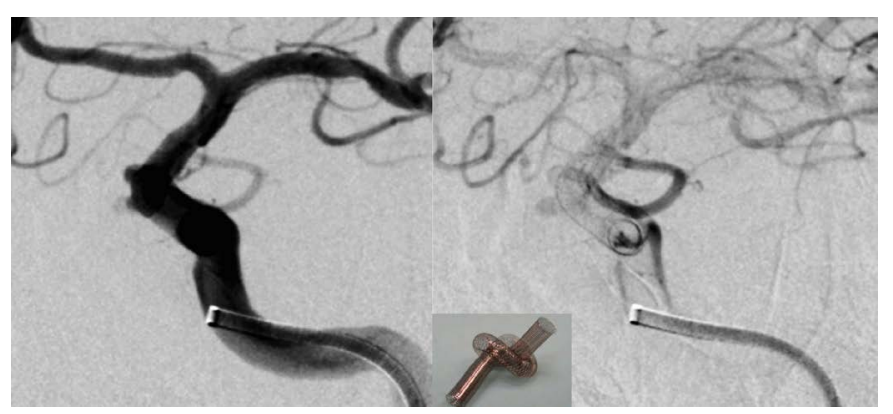

Fig. 4: Digital subtraction angiogram (DSA) shows the flowdiverting stent in the left internal carotid artery.
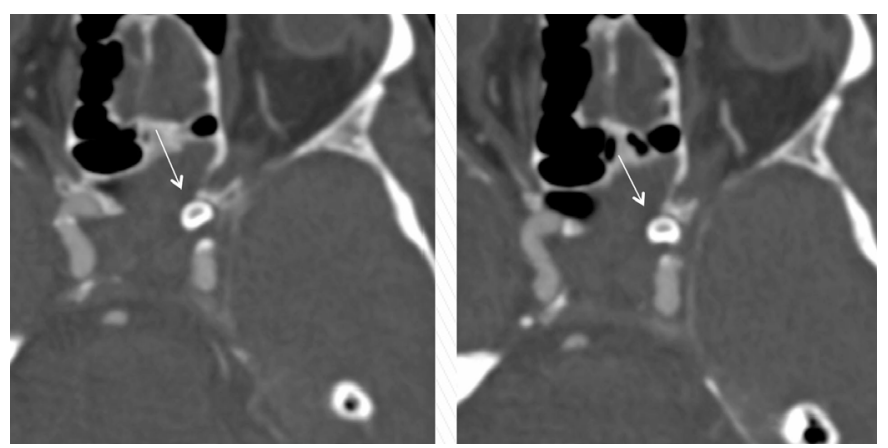

Fig. 5: A follow-up CT angiography obtained three months after endovascular stenting shows successful exclusion of the pseudoaneurysm along the anteromedial margin (arrow).

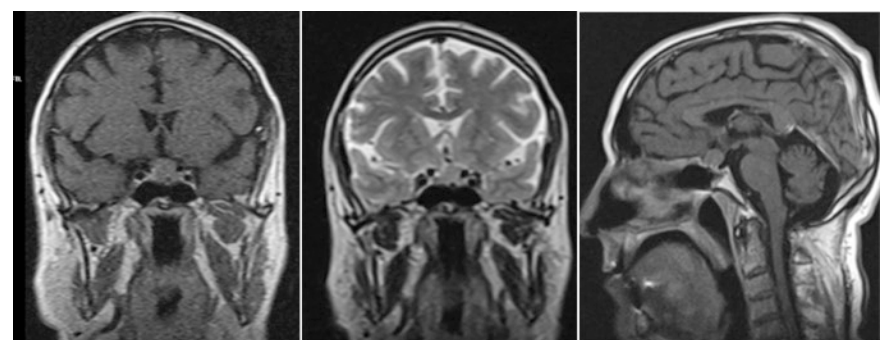

Fig. 6: A follow-up MRI obtained a year after surgery showed adenoma is disappeared.

\section{DISCUSSION}

Vascular injury is one of the most threatening complications associated with transsphenoidal surgery $(2,3)$. Although rare, iatrogenic pseudoaneurysm following endoscopic endonasal pituitary surgery is the most common vascular complication (1). Vascular injury during transsphenoidal resection of pituitary tumors generally occurs during aggressive resection of the tumor by damaging the surrounding vessels (3). These vessels are vulnerable to injury when the sellar dura is opened and the pituitary lesion exposed. As such, damage to the cavernous ICA is the most common type of vascular injury in transsphenoidal surgery (6). In addition to the normal intimate relationship of the ICA with the parasellar region, underlying anatomic variations of the sphenoid bones, an abnormally short distance between ICAs, ICA displacement by the lesion, tumor invasion of the cavernous sinus, tumor adhesion to the ICA, acromegaly with distortion of the local anatomy, and unusual tortuosities in the ICA can all result in an increased risk of vascular injury (8). Previous surgery, previous external radiotherapy (risk may be higher after proton or stereotactic radiation), chemoradiotherapy, or prior treatment with bromocriptine for pituitary tumors have also been linked to ICA injury (4).

Intra-operative ICA rupture creates an immediately challenging surgical field due to a high pressure/high flow hemorrhage scenario, which may rapidly result in exsanguination of the patient. Massive hemorrhage leads to a loss of orientation and an obscured surgical field often resulting in the surgeon blindly attempting nasal packing to control the hemorrhage (4). Packing materials ideally should be placed with just enough force to control the hemorrhage but not to occlude vascular flow (4). Importantly, patients may be at risk for stroke caused by thromboemboli originating in the injured parent artery (7). The vessel injury can also result in pseudoaneurysm formation, even though the artery appears to be structurally intact, packed, and not bleeding (3).

If excessive bleeding occurs during or after transsphenoidal surgery, even after successful intraoperative tamponade, immediate vascular imaging should be obtained to rule out vessel wall damage (3). Various treatment strategies have been used in the management of pseudoaneurysms, including observation (9). Common surgical treatments for pseudoaneurysm, such as clipping, wrapping, trapping, and ligation, are associated with an increased risk of complication or death (3). In our patient, the pseudoaneurysm was successfully treated with preservation of flow within the parent ICA by means of a flow-diverting stent placed across the pseudoaneurysm.

Flow-diverting stents have been proven successful in treatment of ICA aneurysms (10). In general, preserving the ICA using a stent or pipeline is the treatment of choice whenever possible. The safety and effectiveness of stenting are well established (4). Based on such evidence, the use of an endovascular flow-diverting stent was felt to be warranted in this case. Given the success and absence of complications, our results suggest flow-diverting stents may have a role in the treatment of ICA pseudoaneurysms following endoscopic endonasal pituitary surgery (10).

In summary, the presence of either an intraoperative suspicion of vascular injury or any neurologic symptoms and bleeding after transsphenoidal surgery must prompt one to perform rapid angiography to evaluate for vascular injury (11). Delayed complications, such as pseudoaneurysm, could pass unnoticed for some time leading to major morbidity. Therefore, a second angiography may be repeated one or two weeks after the initial intervention for the early detection of these complications; however, the timing for repeat angiography is empirical and not universally accepted. When considering the timecourse, it is important to remember that a pseudoaneurysm can devel- 
op at any point from within hours or even months later (4). Lastly, an endovascular approach to treatment of ICA pseudoaneurysm after transsphenoidal pituitary surgery may be an alternative method with equivalent effectiveness and fewer complications than prior methods. Further studies are needed to confirm this hypothesis in a larger cohort of patients.

\section{REFERENCES}

1. Wilson CB, Dempsey LC. Transsphenoidal microsurgical removal of 250 pituitary adenomas. J Neurosurg 1978; 48(1): 13-22.

2. Cosgrove GR, Villemure JG, Melancon D. Traumatic intracranial aneurysm due to arterial injury at surgery. Case report. J Neurosurg 1983; 58(2): 291-294.

3. Raymond J, Hardy J, Czepko R, Roy D. Arterial injuries in transsphenoidal surgery for pituitary adenoma; the role of angiography and endovascular treatment. AJNR Am J Neuroradiol 1997; 18(4): 655-665.

4. AlQahtani A, Castelnuovo P, Nicolai P, Prevedello DM, Locatelli D, Carrau RL. Injury of the Internal Carotid Artery During Endoscopic
Skull Base Surgery: Prevention and Management Protocol. Otolaryngol Clin North Am 2016; 49(1): 237-252.

5. Chaloupka JC, Putman CM, Citardi MJ, Ross DA, Sasaki CT. Endovascular therapy for the carotid blowout syndrome in head and neck surgical patients: diagnostic and managerial considerations. AJNR Am J Neuroradiol 1996; 17(5): 843-852.

6. Laws ER, Jr. Vascular complications of transsphenoidal surgery. Pituitary 1999; 2 (2): 163-170.

7. Pozzati E, Giuliani G, Poppi M, Faenza A. Blunt traumatic carotid dissection with delayed symptoms. Stroke 1989; 20(3): 412-416.

8. Ciceri EF, Regna-Gladin C, Erbetta A, et al. Iatrogenic intracranial pseudoaneurysms: neuroradiological and therapeutical considerations, including endovascular options. Neurol Sci 2006; 27(5): 317-322.

9. Fleischer AS, Faria MA, Jr., Hoffmann JC, Jr. Pseudoaneurysm complicating superficial temporal artery-middle cerebral artery bypass. Surg Neurol 1979; 12(4): 305-306.

10. Saatci I, Cekirge HS, Ozturk MH, et al. Treatment of internal carotid artery aneurysms with a covered stent: experience in 24 patients with mid-term follow-up results. AJNR Am J Neuroradiol 2004; 25(10): 1742-1749.

11. Cinar C, Bozkaya H, Parildar M, Oran I. Endovascular Management of Vascular Injury during Transsphenoidal Surgery. Interv Neuroradiol 2013; 19(1): 102-109. 